\title{
Correction to: Fourier uniformity of bounded multiplicative functions in short intervals on average
}

\author{
Kaisa Matomäki ${ }^{1}$ - Maksym Radziwiłł ${ }^{2}$. \\ Terence $\mathrm{Tao}^{3}$
}

Published online: 20 November 2019

(C) Springer-Verlag GmbH Germany, part of Springer Nature 2019

\section{Correction to: Invent. math. https://doi.org/10.1007/s00222-019-00926-w}

In the Acknowledgements, the second line should read: MR was supported by NSF grant DMS-1902063 and a Sloan Fellowship.

Publisher's Note Springer Nature remains neutral with regard to jurisdictional claims in published maps and institutional affiliations.

The original article can be found online at https://doi.org/10.1007/s00222-019-00926-w.

$凶$ Maksym Radziwiłł

maksym.radziwill@gmail.com

Kaisa Matomäki

ksmato@utu.fi

Terence Tao

tao@math.ucla.edu

1 Department of Mathematics and Statistics, University of Turku, 20014 Turku, Finland

2 Department of Mathematics, Caltech, 1200 E California Blvd, Pasadena, CA 91125, USA

3 Department of Mathematics, UCLA, 405 Hilgard Ave, Los Angeles, CA 90095, USA 